\title{
Lateral Membrane Waves Constitute a Universal Dynamic Pattern of Motile Cells
}

\author{
Hans-Günther Döbereiner, ${ }^{1, *}$ Benjamin J. Dubin-Thaler, ${ }^{1}$ Jake M. Hofman,${ }^{2}$ Harry S. Xenias, ${ }^{1}$ Tasha N. Sims, ${ }^{3}$ \\ Grégory Giannone, ${ }^{1}$ Michael L. Dustin, ${ }^{3}$ Chris H. Wiggins, ${ }^{4}$ and Michael P. Sheetz ${ }^{1}$ \\ ${ }^{1}$ Department of Biological Sciences, Columbia University, New York, New York 10027, USA \\ ${ }^{2}$ Department of Physics, Columbia University, New York, New York 10027, USA \\ ${ }^{3}$ Skirball Institute of Biomolecular Medicine, New York University School of Medicine, New York, New York 10016, USA \\ ${ }^{4}$ Department of Applied Physics and Applied Mathematics, Columbia University, New York, New York 10027, USA
}

(Received 27 February 2006; published 20 July 2006)

\begin{abstract}
We have monitored active movements of the cell circumference on specifically coated substrates for a variety of cells including mouse embryonic fibroblasts and $T$ cells, as well as wing disk cells from fruit flies. Despite having different functions and being from multiple phyla, these cell types share a common spatiotemporal pattern in their normal membrane velocity; we show that protrusion and retraction events are organized in lateral waves along the cell membrane. These wave patterns indicate both spatial and temporal long-range periodic correlations of the actomyosin gel.
\end{abstract}

DOI: 10.1103/PhysRevLett.97.038102

Directed cell motility requires the coordination and collective movement of cytoskeletal elements. While quite a bit is known about the molecular pathways that regulate actin-based cell motility [1], the mesoscopic behavior of the actomyosin gel is less well understood. Until recently a detailed quantitative characterization of motility phenotypes was lacking; however, progress has now been made in several respects. For example, both experimental [2,3] and theoretical [4] frameworks surrounding the reconstitution of pathogen motility have been established. In addition, a universal description of cell spreading kinetics has been achieved [5], while the physics of active gels has, in general, gained increased attention [6-8]. Furthermore, details concerning the biological function of force sensing and substrate elasticity have been elucidated [9-13]. Finally, detailed flow patterns of the spatial distribution of actin during cell motion have been constructed [14].

In earlier work, we studied the dynamics of the leading membrane edge in spreading mouse embryonic fibroblast cells [15-17] and found several phases characterized by distinct velocity patterns [15]. The main elements of these patterns are regular protrusion and retraction events [16]. Different motile phases have now been seen in epithelial cell protrusions as well [18]. A fundamental biophysical question is how the spatiotemporal organization of these protrusions couples to and results in motility. Previous observations of periodic retractions of the leading membrane edge [17], thought to be central to the durotactic mechanism of the cell, motivated an investigation of spatial correlations. Indeed, we found that protrusion and retraction events are organized in lateral waves along the membrane edge.

High gene similarity in the motile apparatus of both the mouse and the fruit fly [1] suggests that there is an evolutionary advantage to preserve the collective dynamics of the active actomyosin gel underlying cell motility. Thus, one would expect that lateral waves are a quite general phenomenon. Indeed, we observed such waves in two
PACS numbers: 87.17.Jj, 87.15.La, 87.16.Qp

different cell types of the mouse, a mammal, and in one cell type of the fruit fly, an insect. As mice and flies belong to two different phyla of the animal kingdom, the vertebrate subphylus of the chordata and the arthropoda, this suggests an interphylum spatiotemporal membrane velocity pattern within the animal kingdom.

We have monitored active movements of the cell circumference using total internal reflection fluorescence (TIRF) microscopy. Spreading and motility assays were performed for a variety of cells on specifically adhesive substrates, including mouse embryonic fibroblasts and $T$ cells, as well as wing disk cells from fruit flies. Cultured fibroblasts ( RPTP $\alpha+/+)$ and wing disk cells ( $\mathrm{S} 2 \mathrm{R}+$ ) were plated on fibronectin and/or vitronectin coated glass slides, respectively. Primary naïve $T$ cells were derived from the lymph nodes and spleens of mice and allowed to adhere on laterally mobile, supported bilayers containing intercellular adhesion molecules (ICAM-1) and major histocompatibility complex (MHC) molecules with antigenic peptides [19].

The time-lapse sequences of three different motile cells exhibiting lateral wave patterns are shown in Fig. 1. The

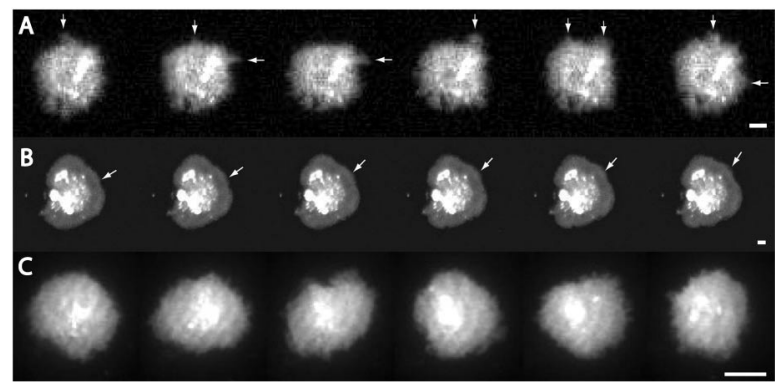

FIG. 1. Time sequences of cell shapes exhibiting lateral membrane waves for mouse embryonic fibroblasts [(a), $\Delta t=30 \mathrm{~s}$ ], fly wing disk cells [(b), $\Delta t=10 \mathrm{~s}$ ], and mouse $T$ cells [(c), $\Delta t=$ $84 \mathrm{~s}$ ] obtained by total internal reflection fluorescence. Arrows point to isolated but highly correlated (a) or propagating (b) protrusions. Bars denote $3 \mu \mathrm{m}$. 

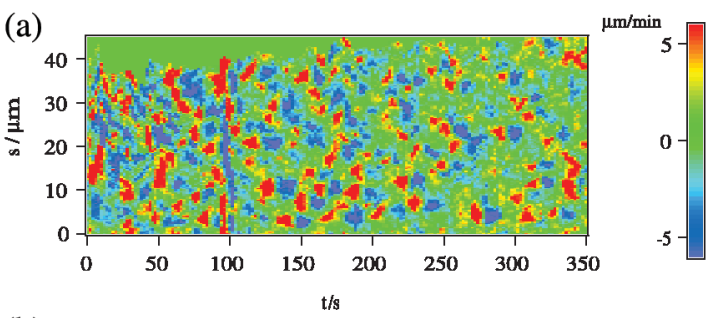

(b)
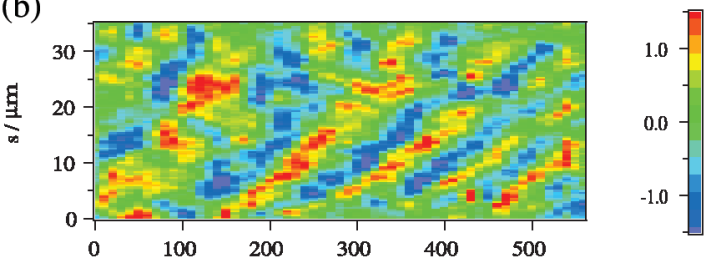

(c)

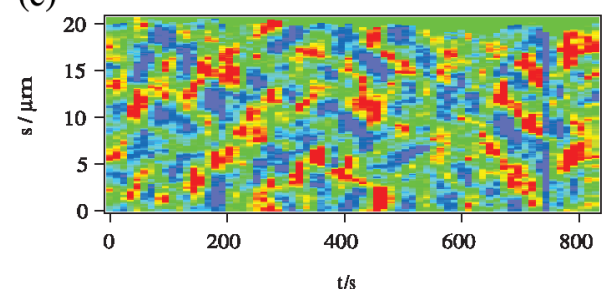

(a)

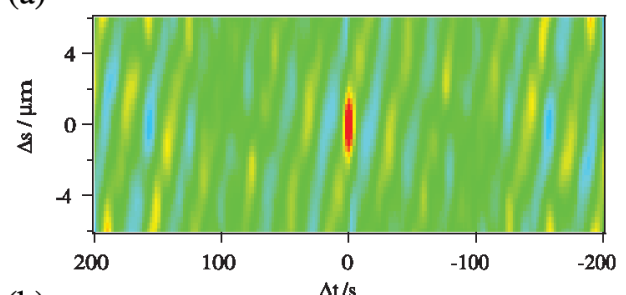

(b)

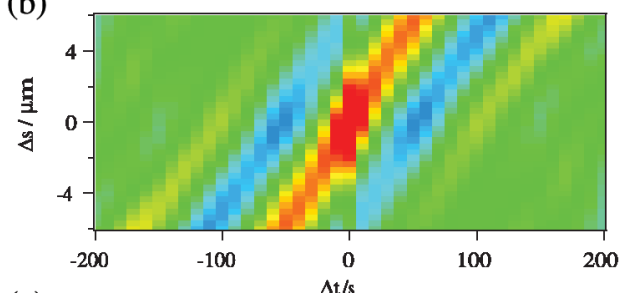

(c)

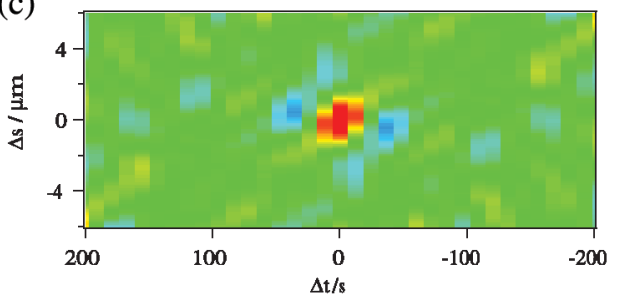

FIG. 2 (color). Normal velocity maps $v_{n}(s, t)$ for mouse embryonic fibroblasts (a), fly wing disk cells (b), and mouse $T$ cells (c). The time resolution is $2 \mathrm{~s}$ (a), $10 \mathrm{~s}$ (b), and $12 \mathrm{~s} \mathrm{(c),}$ respectively. Cell edge velocities are calculated as discussed in the text [see Eq. (1)] from cells shown in Fig. 1. Note the diagonally striped appearance of the velocity map indicating lateral wave patterns.

fibroblast cell is in the prespreading basal phase [15] and periodically extends adhesive protrusions. During extensions and retractions these protrusions show limited, if any, lateral movement. In contrast, the fully spread fly cell exhibits laterally propagating protrusions. Finally, the $T$ cell translates over relatively large distances while periodically undergoing global shape changes.

Analysis of these time-lapse sequences begins with subtraction of the centroid velocity, effectively removing any translational motion of the cell. For each frame, with image coordinates $\mathbf{x}$, a two-component Gaussian mixture model is fit to the pixel intensity distribution using the Expectation Maximation algorithm [20]. From this model, a time-dependent intensity threshold $\bar{h}(t)$ is calculated such that a pixel of intensity $\bar{h}(t)$ is $\alpha$ times as likely to have been drawn from the foreground class (inside the cell) than from the background class (outside the cell). The inside of the cell is segmented from the background and the resulting cell boundary $\Gamma(s, t)$ is parametrized by arclength $s(t)$. This parametrization allows for a time-varying contour length $S(t)$ and accommodates for complex cell morphologies.
FIG. 3 (color). Space-time correlation maps $c(\Delta s, \Delta t)$ as defined in the text, see Eq. (2), for mouse embryonic fibroblasts (a), fly wing disk cells (b), and mouse $T$ cells (c). Correlations are calculated from velocity maps shown in Fig. 2. Bands of high correlation corresponding to lateral waves are clearly visible. The slopes correspond to the respective propagation velocity $v_{L}$, see Table I. In panel (b), the time axis has been reversed to allow direct comparison of slopes among panels.

The normal velocity of each point on $\Gamma(s, t)$ is calculated from gradients of the image data $h(\mathbf{x}(s, t), t)$ as

$$
v_{n}=\frac{\partial_{t}(h(\mathbf{x}, t)-\bar{h}(t))}{|\nabla h(\mathbf{x}, t)|} .
$$

This is equivalent to the kinematic boundary condition in fluid dynamics and a simpler case of the velocity inference problem often addressed by optical flow methods [21].

Color-coded examples of normal velocity maps from the fly and the mouse are shown in Fig. 2. In all cases there are clear bands of consecutive protrusions and retractions. Notably, the bands of positive or negative velocity appear at an angle with respect to the temporal axis, indicating that protrusion or retractions do not happen simultaneously, but rather local protrusion or retraction activity propagates in either direction along the cell edge with a definite speed. These lateral waves are more clearly visible in the corresponding space-time correlation functions (see Fig. 3) defined as

$$
c(\Delta s, \Delta t)=\frac{\int_{0}^{T} \int_{0}^{S(t)}\left(v_{n}(s+\Delta s, t+\Delta t)-\bar{v}_{n}\right)\left(v_{n}(s, t)-\bar{v}_{n}\right) d s d t}{\int_{0}^{T} \int_{0}^{S(t)}\left(v_{n}(s, t)-\bar{v}_{n}\right)^{2} d s d t},
$$


A

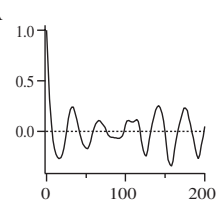

B

$\Delta \mathrm{t} / \mathrm{s}$

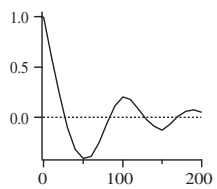

$\Delta t / s$

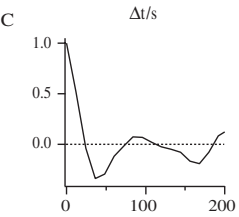

$\Delta t / s$

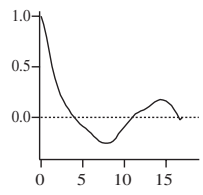

$\Delta \mathrm{s} / \mu \mathrm{m}$

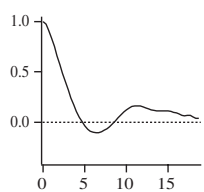

$\Delta \mathrm{s} / \mu \mathrm{m}$

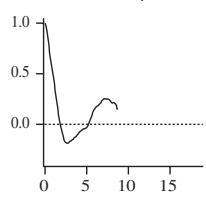

$\Delta \mathrm{s} / \mu \mathrm{m}$

FIG. 4. Spatial $(\Delta t=0)$ and temporal $(\Delta s=0)$ cuts of the correlation maps $c(\Delta s, \Delta t)$ in Fig. 3 for mouse embryonic fibroblasts (a), fly wing disk cells (b), and mouse $T$ cells (c). Numerical characteristics are compiled in Table I.

where $\bar{v}_{n}$ is the mean normal velocity, and we set $v_{n}=\bar{v}_{n}$ outside of the experimental data set. Note that by construction, the space-time correlation function is point symmetric to the origin, i.e., we have $c(\Delta s, \Delta t)=c(-\Delta s,-\Delta t)$. The functional dependence of the equal arclength, $c(0, \Delta t)$, and equal time correlation function, $c(\Delta s, 0)$, respectively, is depicted in Fig. 4, from which the average duration of motility events can be readily obtained. Velocity maps may be consulted for direct comparison. The correlation function crosses zero at $\Delta t_{0}$ corresponding to half the typical duration for protrusion or retraction events. The first minimum at $\Delta t_{\min }$ denotes the time between protrusions and retractions, whereas the second maximum at $\Delta t_{\max }$ correlates with the time between successive protrusions or successive retractions. In general, we find that $\Delta t_{\max } \approx 2 \Delta t_{\min }$, corresponding to a symmetric succession of protrusion and retraction events. From the zero-crossing of the equal time correlation function $c\left(\Delta s_{0}, 0\right)=0$ we obtain the typical spatial extension of protrusions or re-

tractions events. Specific numerical results for the three different cell types studied are listed in Table I.

Cell motility is regulated by various molecular pathways whose stochastic nature is responsible for most of the noise in the normal velocity data. In contrast, the averaging over space and time used in calculating the correlation functions reveals a remarkably regular motility pattern for single cell observations. In fact, ensemble averages would smear out well-defined patterns as each single cell typically is in a slightly different, but equally well-defined configuration due to variations in its environment and history. The characteristic signature of lateral waves of protrusions and retractions is a linear shift of the extrema in the correlation function, given by $\partial c(\Delta s, \Delta t) / \partial \Delta t=0$ (see Fig. 3). From this we find $\Delta s_{\min / \max }=v_{L} \Delta t_{\min / \max }$, where $v_{L}$ is the lateral wave velocity. Extrema are extracted numerically by parabolic fits along the time lag axis for different values of the space lag $\Delta s_{\min / \max }$. We then obtain the wave velocity from the resulting extrema with a linear fit. For all three cell types we find speeds on the order of $100 \mathrm{~nm} / \mathrm{s}$ (see Table I). Our interpretation of the observed motility pattern as waves is reinforced by the result that propagation occurs in both directions with the same velocity as seen most clearly in Fig. 5 for the fly cell.

From a biological perspective, one would like to know the control mechanisms for the lateral waves. In general, the actomyosin gel of motile cells depends on a complex network of diffusive signaling pathways that set various functional interaction parameters. From the observed motility pattern, we can get an estimate of the typical, effective diffusion constant $D_{L}=v_{L} \Delta s_{\max }$ (see Table I). For the fly and the $T$ cell, we find numerical values on the order of $0.1-1 \mu \mathrm{m}^{2} / \mathrm{s}$, as expected for diffusion within the plasma membrane, whereas the fibroblast cells exhibit larger values on the order of $10 \mu \mathrm{m}^{2} / \mathrm{s}$, as expected for bulk diffusion. Thus, we conclude that the control mechanisms effective in the fly and the $T$ cell are likely to involve membrane bound signaling cascades. Indeed, the immune response of $T$ cells crucially depends on cluster formation in the membrane [22]. In contrast, bulk cytoplasmic control mechanisms dominate for the fibroblasts. It is not clear at present whether wave propagation involves motor activity

TABLE I. Numerical characteristics of correlation functions depicted in Fig. 4 as defined in the text. Lateral wave velocities $v_{L}$ and derived effective diffusion constants $D_{L}$ are given for each cell line.

\begin{tabular}{cccc}
\hline \hline & Mouse $\left(\mathrm{RPTP} \alpha_{+/+}\right)$ & Fly $(\mathrm{S} 2 \mathrm{R}+)$ & Mouse $(T$ cells $)$ \\
\hline$\Delta t_{0}$ in $s$ & $7 \pm 2$ & $28 \pm 10$ & $30 \pm 12$ \\
$\Delta t_{\min }$ in $s$ & $16 \pm 2$ & $55 \pm 10$ & $46 \pm 12$ \\
$\Delta t_{\max }$ in $s$ & $33 \pm 2$ & $107 \pm 10$ & $88 \pm 12$ \\
$\Delta s_{0}$ in $\mu \mathrm{m}$ & $4.0 \pm 0.3$ & $4.7 \pm 0.3$ & $1.8 \pm 0.1$ \\
$\Delta s_{\min }$ in $\mu \mathrm{m}$ & $8.0 \pm 0.3$ & $6.3 \pm 0.3$ & $2.6 \pm 0.1$ \\
$\Delta s_{\max }$ in $\mu \mathrm{m}$ & $14.3 \pm 0.3$ & $11.0 \pm 0.3$ & $7.3 \pm 0.3$ \\
$v_{L}$ in $\mathrm{nm} / \mathrm{s}$ & $400 \pm 30$ & $120 \pm 10$ & $55 \pm 5$ \\
$D_{L}$ in $\mu \mathrm{m}^{2} / \mathrm{s}$ & $5.7 \pm 0.6$ & $1.3 \pm 0.1$ & $0.4 \pm 0.05$ \\
\hline \hline
\end{tabular}




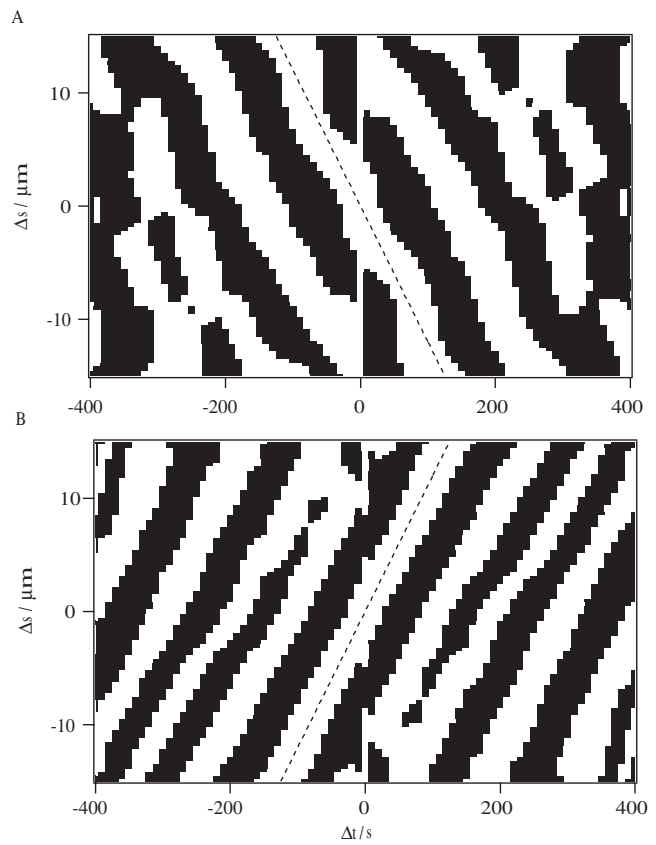

FIG. 5. Binary correlation maps of the fly cell depicted in Fig. 1(b). Correlation maps were calculated as sign $[c(\Delta s, \Delta t)]$ in two different regions of the cell perimeter. Lateral waves are found to move in opposite directions with velocities $v_{L}=$ $\pm 120 \mathrm{~nm} / \mathrm{s}$, as indicated by the dashed lines.

or is purely actin related; see, however, the discussion of lateral waves in endothelial cells by Machacek and Danuser [18].

We have presented a first quantitative analysis suggestive of interphylum motility phenotypes in the animal kingdom. The developed velocity calculation and correlation function analysis represent powerful tools for quantifying cell motility and offer distinct advantages over the coarse-grained histograms and semiquantitative kymographs usually encountered in the literature. We envision this analysis of phenotypes as a useful, general method to disentangle complex, collective cell behavior and hope it will spur the development of two-dimensional theoretical models of active gels. Future work will focus on the dependence of wave patterns on cellular phases [15] and their control by functional protein modules $[23,24]$.

*Electronic address: hgd@ biophysik.uni-bremen.de
[1] T. D. Pollard and G. G. Borisy, Cell 112, 453 (2003).

[2] D. Pantaloni, C. Le Clainche, and M-F. Carlier, Science 292, 1502 (2001).

[3] A. Bernheim-Grosswasser, S. Wiesner, R. M. Golsteyn, M.-F. Carlier, and C. Sykes, Nature (London) 417, 308 (2002).

[4] F. Gerbal, P. Chaikin, Y. Rabin, and J. Prost, Biophys. J. 79, 2259 (2000).

[5] F. Chamaraux, S. Fache, F. Bruckert, and B. Fourcade, Phys. Rev. Lett. 94, 158102 (2005).

[6] D. Humphrey, C. Duggan, D. Saha, D. Smith, and J. Käs, Nature (London) 416, 413 (2002).

[7] K. Kruse and F. Jülicher, Phys. Rev. E 67, 051913 (2003).

[8] K. Kruse, J.F. Joanny, F. Jülicher, J. Prost, and K. Sekimoto, Eur. Phys. J. E 16, 5 (2005).

[9] N.Q. Balaban et al., Nature Cell Biology 3, 466 (2001).

[10] G. Jiang, G. Giannone, D. R. Critchley, E. Fukumoto, and M. P. Sheetz, Nature (London) 424, 334 (2003).

[11] I. B. Bischofs, S. A. Safran, and U.S. Schwarz, Phys. Rev. E 69, 021911 (2004).

[12] A. Engler, L. Bacakova, C. Newman, A. Hategan, M. Griffin, and D. Discher, Biophys. J. 86, 617 (2004).

[13] D. E. Discher, P. Janmey, and Y.-l. Wang, Science 310, 1139 (2005).

[14] A. Ponti, M. Machacek, S. L. Gupton, C. M. WatermanStorer, and G. Danuser, Science 305, 1782 (2004).

[15] H.-G. Döbereiner, B. Dubin-Thaler, G. Giannone, H. S. Xenias, and Michael P. Sheetz, Phys. Rev. Lett. 93, 108105 (2004).

[16] B. Dubin-Thaler, G. Giannone, H.-G. Döbereiner, and M. P. Sheetz, Biophys. J. 86, 1794 (2004).

[17] G. Giannone, B. Dubin-Thaler, H.-G. Döbereiner, and M. P. Sheetz, Cell 116, 431 (2004).

[18] M. Machacek and G. Danuser, Biophys. J. 90, 1439 (2006).

[19] A. Grakoui, S. K. Bromley, C. Sumen, M. M. Davis, A. S. Shaw, P. M. Allen, and M. L. Dustin, Science 285, 221 (1999).

[20] J. Kittler and J. Illingworth, Pattern Recognition 19, 41 (1986).

[21] T. Poggio, V. Torre, and C. Koch, Nature (London) 317, 314 (1985).

[22] G. Campi, R. Varma, and M. L. Dustin, Experimental Biology and Medicine 202, 1031 (2005).

[23] M.P. Sheetz, B. J. Dubin-Thaler, G. Giannone, G. Jiang, and H.-G. Döbereiner, in Cell Migration, edited by D. Wedlich (Wiley, New York, 2005).

[24] H.-G. Döbereiner, B. J. Dubin-Thaler, G. Giannone, and M. P. Sheetz, J. Appl. Physiol. 98, 1542 (2005). 